\title{
Heart Sounds Classification using Feature Extraction of Phonocardiography Signal
}

\author{
Mandeep Singh \\ Electrical \& Instrumentation Engineering \\ Department \\ Thapar University \\ Patiala, India.
}

\author{
Amandeep Cheema \\ Electrical \& Instrumentation Engineering \\ Department \\ Thapar University \\ Patiala, India.
}

\begin{abstract}
The Phonocardiogram (PCG) signals contain very useful information about the condition of the heart. By analyzing these signals, early detection and diagnosis of heart diseases can be done. It is also very useful in the case of infants, where ECG recording and other techniques are difficult to implement. In this paper, a classification method is proposed to classify normal and abnormal heart sound signals having murmurs without getting into the cumbersome process of segmenting fundamental heart sounds (FHS) using Electrocardiogram (ECG) gating. The proposed algorithm can be easily implemented on latest electronic stethoscopes, and therefore the unnecessary ECG can be avoided.
\end{abstract}

\section{General Terms}

Classification algorithm.

\section{Keywords}

Heart sounds, Murmurs, Feature extraction, Naïve Bayes, Bayes Net classifier.

\section{INTRODUCTION}

Heart disease is a major health problem and a leading cause of fatality throughout the world. The treatment can be easier, efficient and economical if the condition is detected early. So it would be very beneficial to detect heart diseases at an early stage. Cardiac disorders can be detected efficiently and economically using auscultation as it requires minimal equipment. Sometimes this is the only available option for diagnosis as in case of primary health care centres, where other high-end instruments for diagnosis are unavailable and also in case of infants where other techniques like ECG are difficult to implement. Conventional auscultation requires extensive training and experience and storage of records for follow ups and future references is not possible [1]. This is the driving force for this study in order to move towards automatic auscultation using electronic stethoscopes. Heart sounds comprise of four components among which the dominant two S1 and S2 (see Figure 1) are commonly known as FHS and are caused by closing of valves. After that S3 is caused by the vibration of ventricular valves as a result of first rapid filling, S4 occurs when atria contract during second phase of ventricular filling. The low-pitched S1 is best heard at mitral auscultation site as it is caused by closure of mitral and tricuspid valves and shorter duration but louder sound S2 is caused by aortic and pulmonary valve closure and is best heard over aortic auscultation area [2].
The aim of this study is to develop a method for classification of heart sounds into normal and abnormal sounds so that state of heart could be periodically checked at home and everybody doesn't have to wait for symptoms of disease to appear and then approach a cardiologist. The PCG signals can be an early indicator to heart problems so before worsening of the problem, a proper diagnosis can be done. Then, the other techniques like Echocardiography could be implemented to get a better view of problem. So, the proposed method is not a replacement of other techniques but an early indicator to the problem in order to prevent worsening of the situation. In this study the PCG signals were analyzed for normal and murmur heart sounds and established that between the fundamental heart sounds S1 and S2, the amplitude of the murmur signal is higher than normal signal (see Figure 2). Then the segments between S1 and S2 of same cardiac cycle, S2 of one cardiac cycle and S1 of next cardiac cycle were selected for further analysis. The features were extracted in order to differentiate between normal and murmur signals.

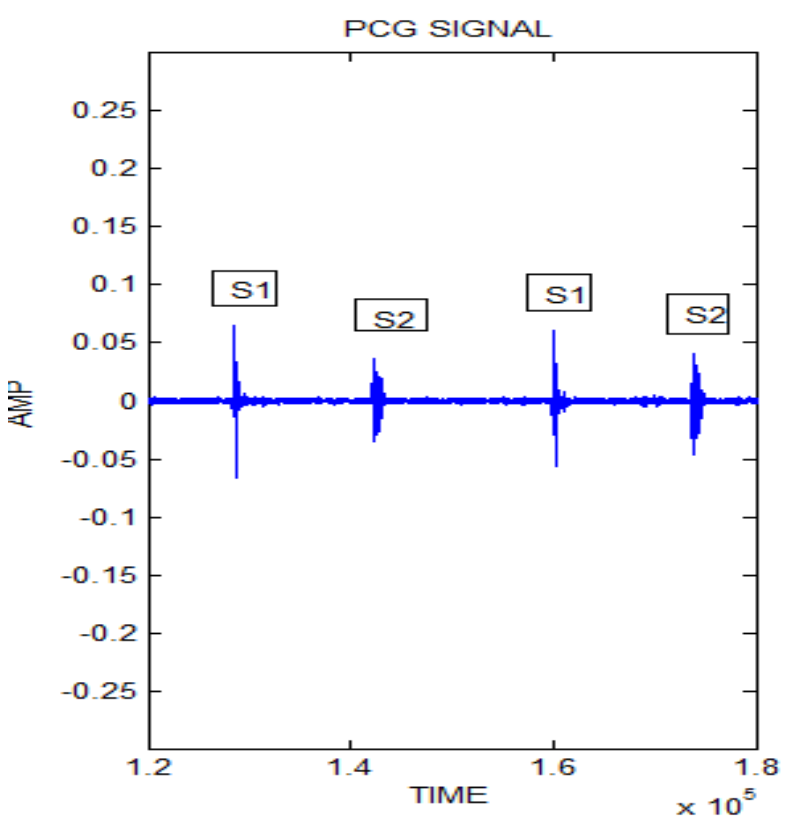

Figure 1: PCG Signal showing S1 and S2 

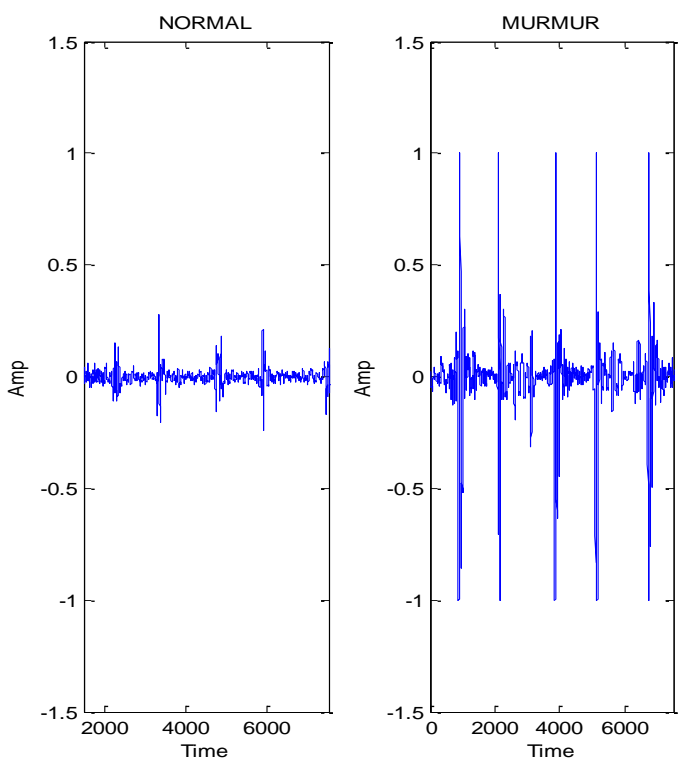

Figure 2: Amplitude differences in Normal and Murmur Signals

\section{PREVIOUS WORK}

The classification of heart sounds is not a new topic, however it is still in a developing stage as far as its embedded applications are concerned. With the use of latest technology, mobile phones can also be used as an electronic stethoscope and the digital phonocardiogram signals can be easily acquired, saved and transmitted to a cardiologist for further analysis. Most of the research in the auscultation field is seen mainly till 1980s but due to other methods like Echocardiography, research trends in PCG decreased, but in past few years it has shown a boost due to improvements in personal computers and signal processing techniques [3-4]. The abrupt frequency changes, the complex and highly nonstationary nature of the heart sound signals make the heart sound signal analysis a tedious job. The FFT and wavelet approaches have been applied to this in various studies and the work of correlating the heart sounds with the heart defects has been done [5-10]. Some related works to this study are as follows:

Muruganantham, (2003) derived various features like average power, total power, mean power frequency, median frequency, frequency variance, frequency skewness, frequency kurtosis, and jitter from frequency domain [11]. Shui et al. (2004) used wavelet analysis for feature extraction in order to distinguish between normal and aortic stenosis patients. Simultaneous recordings of PCG and ECG were done and ECG acts as a guide to characterize heart sounds [12]. Segaier et al. (2005) used STFT (Short Time Fourier Transform) for characterization of systolic murmur [13]. Jiang et al. (2006) extracted cardiac sound characteristic waveforms (CSCW) from the cardiac sounds recorded by an electric stethoscope. The diagnostic parameters are [T1, T2, T11, T12] where $\mathrm{T} 1$ and $\mathrm{T} 2$ are the widths of the first sound $\mathrm{S} 1$ and the second sound S2, T11 is the time interval between two abutted S1, which indicates the heart beat rhythm condition and T12 is the time interval between $\mathrm{S} 1$ and $\mathrm{S} 2$, which is an indicator to express the heart valvular murmurs [14]. Ahlstrom C et al. (2005) [15] and Ahlstrom C et al. (2006) used tool to be able to investigate how signal content varies over time. Stockwell's TFR formed the basis of this work. Shannon energy was used to measure intensity and a wavelet detail was used to measure intensity in a certain frequency interval. Recurrence points of the first kind, T1, are used to locate S1 and S2 after which S3 is sought in time windows 100-300 ms after the two heart sounds [16]. Noponen et al. (2007) combined spectrogram and traditional phonocardiogram to distinguish innocent murmurs from pathological murmurs [17]. Amit et al. (2009) worked on the basis of hierarchical clustering, compact data representation in the feature space of cluster distances [18]. Maglogiannisa et al. (2009) proposed a diagnosis system using SVM (Support Vector Machine) to classify heart valve disease [19].

Mostly the studies done so far use simultaneous ECG recordings, termed as ECG gating for the segmentation of FHS and then classification of signals is done. This is a cumbersome method and additional ECG equipment is required, thus making it an expensive method. Sometimes a tedious pre-processing of signals has to be done as depicted in various studies. This is the driving force of this study. The method presented in this paper is simple and cost-efficient as it does not require ECG gating. The equipment required for this method is minimum i.e. an electronic stethoscope and a PC/laptop.

\section{MATERIALS AND METHODOLOGY 3.1 Signal Acquisition}

The PCG signal acquisition can be done by an electronic stethoscope which responds to the sound waves identically to the conventional acoustic stethoscope with the changes in electric field replacing the changes in air pressure. The electronic stethoscopes being very expensive and not readily available in developing countries Shervegar et al. (2011) made a very low-cost electronic stethoscope using electronic chest-piece, the amplifier circuit and a PC/laptop [20]. However for this study, an electronic database of PCG signals is taken from:

http://www.peterjbentley.com/heartchallenge/index.html [21]. The dataset used is taken from a clinic trial in hospitals using the digital stethoscope DigiScope ${ }^{\circledR}$.

In this study a dataset recorded from Phonocardiograph having 60 signals is used out of which 30 are normal signals and 30 are murmur signals.

\subsection{Feature Extraction}

In this study a total of 23 features from time domain, frequency domain, cepstrum and statistical features were extracted that could have potential to discriminate among the normal and murmur signals. This study uses the common assumption that systole is shorter than diastole. Unlike other studies, the features in the whole signal as well as separately in systolic and diastolic regions have been extracted in order to deal with the situations of systolic and diastolic murmur. The features are enlisted in the Table 1.

In the terminology used the suffix 1 shows that the feature is extracted in the systole and the suffix 2 shows that the feature is extracted in the diastole.

Peak Amplitude 1= the maximum amplitude in systolic period.

$\mathrm{t} 1=$ time duration of $\mathrm{S} 1$

$\mathrm{t} 2=$ time duration of $\mathrm{S} 2$

$\mathrm{t} 12=$ time duration from end of S1 to start of S2

$\mathrm{t} 21=$ time duration from end of S2 to start of next cycle's S1. 
Table 1: List of Features Extracted for Classification

\begin{tabular}{|c|c|c|c|}
\hline $\begin{array}{l}\text { S. } \\
\text { No }\end{array}$ & Feature & Feature domain & Source \\
\hline 1 & Peak Frequency & Frequency domain & [11] \\
\hline 2 & Peak Amplitude & Time domain & [18] \\
\hline 3 & Total Power & Time domain & [11] \\
\hline 4 & $\begin{array}{c}\text { Total Harmonic } \\
\text { Distortion (THD) }\end{array}$ & Frequency domain & \\
\hline 5 & Bandwidth & Frequency domain & [11] \\
\hline 6 & Q-Factor & Frequency domain & \\
\hline 7 & $\begin{array}{l}\text { Cepstrum Peak } \\
\text { Amplitude }\end{array}$ & Cepstrum & \\
\hline 8 & Peak Frequency 1 & Frequency domain & \\
\hline 9 & Peak amplitude 1 & Time domain & \\
\hline 10 & Total Power 1 & Time domain & \\
\hline 11 & Bandwidth 1 & Frequency domain & \\
\hline 12 & Q-Factor 1 & Frequency domain & \\
\hline 13 & Peak Frequency 2 & Frequency domain & \\
\hline 14 & Peak Amplitude 2 & Time domain & \\
\hline 15 & Total Power 2 & Time domain & \\
\hline 16 & Bandwidth 2 & Frequency domain & \\
\hline 17 & Q-Factor 2 & Frequency domain & \\
\hline 18 & Mean 12 & Statistical & Proposed \\
\hline 19 & $\begin{array}{l}\text { Zero Crossing } \\
\text { Rate (ZCR) }\end{array}$ & Time domain & [22] \\
\hline 20 & $\mathrm{t} 1$ & Time domain & [14] \\
\hline 21 & $\mathrm{t} 2$ & Time domain & [14] \\
\hline 22 & $\mathrm{t} 12$ & Time domain & [14] \\
\hline 23 & $\mathrm{t} 21$ & Time domain & [14] \\
\hline
\end{tabular}

The potential seen in these features and hence the reason for evaluating these features have been explained further.

Peak Frequency: It shows the frequency at which the peak amplitude occurs. Since the murmurs and normal signals vary in amplitude and frequency, it could have been a potential feature.

Peak Amplitude: It shows the peak value of the signal. The murmur and normal signals vary in amplitude so this feature had potential. A higher Peak amplitude values are expected for murmur signals as compared to normal signals.

Total Power: It shows the total power of the signal. The murmur is a higher amplitude signal and hence is expected to have a higher value of this feature.

Total Harmonic Distortion (THD): It is the measurement of the harmonic distortion present in the signal and is defined as the ratio of the sum of the powers of all harmonic components to the power of the fundamental frequency. A comparatively higher THD is expected for murmur signals.

Bandwidth: It is the difference between the upper and lower frequencies in a continuous set of frequencies. As murmurs are high in frequency so a higher bandwidth is expected in case of murmur signals.

Q-Factor: A higher Q-Factor indicates that oscillations die out more slowly. So, a higher Q-Factor is expected in case of murmur signals.

Cepstrum Peak Amplitude: The main information is concentrated in the starting of cepstrum and we observed the peak values at the start. Hence Cepstrum Peak Amplitude is evaluated as a potential feature.

Mean 12: In most of the murmur signals visible differences were seen in the regions between $\mathrm{S} 1$ and $\mathrm{S} 2$ or $\mathrm{S} 2$ and next cycle's S1. So, the mean was found in these regions and maximum of these two values is considered as Mean12. This is done in order to explore both the possibilities i.e. systolic murmur and diastolic murmur. A higher Mean 12 is expected for murmur signals.

Zero Crossing Rate (ZCR): The ZCR is the rate of signchanges along a signal, i.e., the rate at which the signal changes from positive to negative or back. A larger value of ZCR is expected for murmur signals.

t1: It is the time duration of S1. Normally, S1 are shorter duration sounds but in case of murmurs considerably longer $\mathrm{S} 1$ could be expected. This is because murmurs could appear just after S1 and can make S1 look longer than normal S1 sound.

t2: It is time duration of S2. It is longer than S1. Considerably a longer S2 could be expected for murmur signal than for the normal signal. This is because the murmur could appear just after S2 to make it look longer for murmur signals.

t12: It is the time duration from end of S1 to start of S2. As explained earlier that a murmur could appear just after S1 and hence the region between the end of S1 to start of S2 could become smaller. So, in case of murmurs smaller t12 is expected.

t21: It is the time duration from end of S2 to start of next cycle's S1. As the murmur could appear just after S2 hence the region between the end of S2 to start of next cycle's S1 would look smaller.

\subsection{Feature Selection and Classification}

All the features are calculated from the PCG signals. First of all Ranker and Info Gain Attribute Evaluation is applied on the feature set to find the most significant features. In the feature selection phase finally 5 optimal features were selected. Subsequently, Bayes Net, Naïve Bayes and some other classifiers are used for classification. The selected optimal features are enlisted in Table 2.

Table 2: List of Selected Features for Classification

\begin{tabular}{|c|c|c|}
\hline $\begin{array}{c}\text { S. } \\
\text { No. }\end{array}$ & Feature & Feature domain \\
\hline 1. & Total Power 1 & Time domain \\
\hline 2. & Q-Factor 1 & Frequency domain \\
\hline 3. & $\mathrm{t} 1$ & Time domain \\
\hline 4. & $\mathrm{t} 12$ & Time domain \\
\hline 5. & Mean12 & Statistical \\
\hline
\end{tabular}

These 5 optimal features are enough to segment the signals as normal and murmur signals. The method presented thus requires evaluating these 5 features of a signal for classifying it as normal and murmur signal.

\section{RESULTS}

In this paper some significant features have been introduced. By using these features the classification accuracy improved in case of various classifiers as shown in Table 3. So, these new features proved to be very efficient for classification of normal and murmur signals. This paper also proposes the approach of evaluating some already existing features in both the systolic and diastolic regions in order to deal with the situations of systolic and diastolic murmurs.

In this study, 5 folds cross-validation is used for results. Various classifiers were used in this study in order to find out the best classifier that suits the problem. 
Table 3: Classification Accuracy with and without using new features

\begin{tabular}{|c|c|c|c|c|}
\hline Classifier & $\begin{array}{c}\text { TP } \\
\text { Rate } \\
(\mathbf{N})\end{array}$ & $\begin{array}{c}\text { TP } \\
\text { Rate } \\
(\mathbf{M})\end{array}$ & $\begin{array}{c}\text { Accuracy } \\
(\%) \\
\text { without } \\
\text { these new } \\
\text { features }\end{array}$ & $\begin{array}{c}\text { Accuracy } \\
(\%) \\
\text { with } \\
\text { these new } \\
\text { features }\end{array}$ \\
\hline $\begin{array}{c}\text { Bayes } \\
\text { Net }\end{array}$ & 0.933 & 0.9 & 86.6667 & 91.6667 \\
\hline $\begin{array}{c}\text { Naïve } \\
\text { Bayes }\end{array}$ & 0.933 & 0.933 & 88.3333 & 93.3333 \\
\hline SGD & 0.967 & 0.867 & 90 & 91.6667 \\
\hline $\begin{array}{c}\text { Logit } \\
\text { Boost }\end{array}$ & 0.933 & 0.833 & 83.3333 & 88.3333 \\
\hline
\end{tabular}

The improvement in classification efficiency using new features is evident. Naïve Bayes proved to be the best suited classifier for this application. It gave the highest accuracy amongst all other classifiers used. Hence Naïve Bayes classifier is used because the aim is to minimize the probability of murmur being classified as normal signal. The results of classification using Naïve Bayes classifier are shown in Table 4

Table 4: Confusion Matrix

\begin{tabular}{|c|c|c|c|}
\hline Classifier & Normal & Murmur & \\
\hline \multirow{2}{*}{ Naïve Bayes } & 28 & 2 & Normal \\
\cline { 2 - 4 } & 2 & 28 & Murmur \\
\hline
\end{tabular}

The efficiency parameters for the feature extraction methodology, evaluated from the confusion matrix using Naïve Bayes classifier are as shown in Table 5.

\section{Table 5: Efficiency Parameters for Feature Extraction Methodology}

\begin{tabular}{|c|c|}
\hline Parameter & Value \\
\hline Accuracy & $28+28 / 28+2+28+2 * 100=93.33 \%$ \\
\hline Sensitivity & $28 / 28+2 * 100=93.33 \%$ \\
\hline Specificity & $28 / 28+2 * 100=93.33 \%$ \\
\hline
\end{tabular}

The efficiency parameters achieved are high and thus can lead to more reliable diagnosis.

\section{CONCLUSION}

The method proposed in this study is cost efficient as it requires minimal equipment and does not require ECG gating. The method shown in this study can easily be implemented in the existing electronic stethoscope by interfacing it with the present embedded technology. The proposed method uses only 5 features for heart sounds classification with the accuracy of $93.33 \%$. In future, the proposed method can also be implemented in latest mobile phones and can be used for early detection of some common heart diseases. This method would be extremely useful for the developing countries and for rural health management as only the electronic stethoscope with embedded technology is required in this method.

\section{ACKNOWLEDGMENTS}

Authors are thankful to Bentley, P., Nordehn, G., Coimbra, M., and Mannor, S. for PCG dataset from "The PASCAL Classifying Heart Sounds Challenge 2011.”

\section{REFERENCES}

[1] Roy, D., Sargeant, J., Gray, J., Adn, B., Allen, M., and Fleming, M. 2002. Helping family physicians improve their cardiac auscultation skills. Journal of Continuing Education in the Health Professions 22. 152-159.

[2] Babaei, S., and Geranmayeh, A. 2009. Heart Sound reproduction based on neural network classification of cardiac valve disorders using wavelet transforms of PCG signals. Elsevier. Computers in Biology and Medicine 39. 8-15.

[3] Kagawa, Y., Sato, N., Nitta, S., Saji, K., Tanaka, M., Shlbota, Y., and Horiuchi, T. 1977. Sound Soectroanalvtic Diagnosis of Malfunctionning Prosthetic Valves. Tokohu J. Exp. Med., 123. 77-89.

[4] Durand, L., Blanchard, M., Sabbah, H., Hamid, M., Kemp S., and Stein, P. 1988. A Bayes model for automatic detection and quantification of bioprosthetic valve degeneration. Mathematical and Computer Modelling, Vol. 11. 158-163.

[5] Barschdor, D., Femmer, U., and Trowitzsch, E. 1995 Automatic phonocardiogram signal analysis in infants based on wavelet transforms and artificial neural networks. Computers in Cardiology. IEEE, Vienna, Austria. 753-756.

[6] Asir, B., Khadra, L., Abbasi, A., and Mohammed, M. 1996. Time-frequency analysis of heart sounds. Proc. of IEEE TENCON Conf. on Dig. Sig. Proc. Appl., vol. 2, Perth, WA, Australia. 553-558.

[7] Asir, B., Khadra, L., Abbasi A., and Mohammed, M. 1996. Multiresolution analysis of heart sounds. Proceedings of the Third IEEE Internationall Conf. on Elec., Circ., and Sys., vol. 2, Rodos, Greece. 1084-1087.

[8] Lee, J., Lee, S., Kim, I., Min, H., and Hong, S. 1999. Comparison between short time Fourier and wavelet transform for feature extraction of heart sound. Proceedings of IEEE TENCON 99, vol. 2, Cheju Island, South Korea. 1547-1550.

[9] Shino, H., Yoshida, H., Mizuta H., and Yana, K. 1997. Phonocardiogram classification using time-frequency representation. Proc. of the 19th International Conf. of the IEEE Eng. in Med. and Biol. Soc., vol. 4, Chicago, IL. $1636-1637$.

[10] Rangayyan, R., and Lehner, R. 1988. Phonocardiogram Signal Analysis: A Review. CRC Critical Reviews in Biomedical Engineering, 15(3). 211-236.

[11] Muruganantham. 2003. Methods for Classification of Phonocardiogram. TENCON.

[12] Shui, L. 2004. AM13 Analysis of Heart Sound. Thesis. National University of Singapore.

[13] Segaier, M., Lilja, O., Lukkarinen, S., Ornmo, L., Sepponen, R., and Pesonen, E. 2005. Computer-Based Detection and Analysis of Heart Sound and Murmur. Annals of Biomedical Engineering, Vol. 33, No. 7. 937942.

[14] Jiang, Z., and Choi, S. 2006. A cardiac sound characteristic waveform method for in-home heart disorder monitoring with electric stethoscope. Expert Systems with Applications 31. 286-298.

[15] Ahlstrom, C., Liljefelt, O., Hult P., and Ask, P. 2005. Processing of the Phonocardiographic Signal, Methods 
for the Intelligent Stethoscope. Studies in Science and Technology. Thesis No. 1253.

[16] Ahlstrom, C., Hult, P., and Ask, P. 2006. Thresholding distance plots using true recurrence points. Proceedings of the IEEE Conference on Acoustics, Speech and Signal Processing.

[17] Noponen, A., Lukkarinen, S., Angerla, A., and Sepponen, R. 2007. Phono-spectrographic analysis of heart murmur in children. BMC Pediatrics.

[18] Amit, G., Gavriely, N., and Intrator, N. 2009. Cluster analysis and Classification of Heart Sounds. Elsevier, Biomedical Signal Processing and Control 4. 26-36.

[19] Maglogiannis, I., Loukis, E., Zafiropoulos, E., and Statis, A. Support Vector Machine-based identification of heart valve diseases using Heart Sounds. Elsevier. Computer methods and programs in Biomedicine 95. 47-61.

[20] Vishwanath, M., Shervegar, Bhat, G., and Shetty, R. 2011. Phonocardiography- the future of cardiac auscultation. International Journal of Scientific \& Engineering Research Volume 2, Issue 10.

[21] Bentley, P., Nordehn, G., Coimbra, M., Mannor, S. 2011. The PASCAL Classifying Heart Sounds Challenge 2011. www.peterjbentley.com/heartchallenge/index.html.

[22] Rabiner, L., and Shafer, R. 2008. $2^{\text {nd }}$ Edition. Digital Processing of Speech Signals. Prentice Hall: New Jersey, USA. 